\title{
An experimental study on the application of Maths transformation thoughts in College Mathematics Teaching
}

\author{
Xu chongkun ${ }^{1,}$, , Sun xiaoying ${ }^{2}$ and Duan xiaofang ${ }^{3}$ \\ 1,2,3 Nanchang Institute of Science and Technology, 330108 \\ axuchongkunjx@126.com
}

\begin{abstract}
Keywords: Domestication; College mathematics; Mathematics teaching; Method application; Experimental study.
\end{abstract}

\begin{abstract}
As one of the basic thought of the idea of university mathematics, almost throughout the entire university mathematics content and many mathematical methods, and meet the students' thinking ability and their real life experience is highly valued by the people for mathematics education. In the classroom teaching of mathematics, the use of thought can improve teaching efficiency and cultivate students' ability of mathematical thinking, and improve the level of teaching and learning of college mathematics. Paper the basic meaning of the idea, in the analysis of interaction and learning in mathematics teaching; summarizes the related research from two aspects of horizontal and vertical; based on the research on the principle and strategy of the idea of philosophical foundation, combined with the mathematics teaching practice, through experimental research, analysis of the use of the idea in the university mathematics teaching effect. Summarize the effective way to use the high school mathematics teaching ideas: the first is to improve the knowledge system, the attention in the teaching stage "different topics combined with the usual infiltration", and put this idea into the new teaching organic knowledge, problem-solving training and summary of the review, to avoid the negative effects of excessive attention again to bring. The teaching of college mathematics teaching consciously of mathematical thinking, to promote the study of mathematics teaching, improve the quality of teachers and students of mathematics innovation ability, promote the application of mathematics in other areas, has a very positive and far-reaching significance..
\end{abstract}

\section{Introduction}

In today's rapid development of the information technology revolution era, many domestic and foreign authoritative scientists predict that, in the new century, "mathematics is the science and technology and economic development of the key" in local competition, mathematics is often the magic weapon of winning the final, the quality of mathematics education is directly related to the training of technical personnel and the quality of the whole people to improve. As the first to study mathematics in front of elementary education teachers, the essence of mathematics, and on the basis of this study of teaching methods, not only focus on local knowledge and problem-solving skills, more should be thinking and methods of mathematics, the individual will be thorough understanding as imparting knowledge of the main line[1], let the students not only learn knowledge but also to master the idea and method to teach students' mathematics thinking, mathematics become a kind of life attitude with their life. Mathematics thought is refers to some concrete mathematics understanding process to enhance the correct viewpoint, in the subsequent understanding activity repeatedly uses and the confirmation has the universal significance and the relatively stable characteristic. Mathematics thought is the essence of the concept, method and theory of mathematics. Because of this, the thought of mathematics has become the guiding ideology to set up the theory of mathematics and solve the problem of mathematics. It should not be confused with mathematical thought and mathematical thinking, the latter is more inclined to "show that" the human brain and mathematical object interaction, rational thinking activities in accordance with the understanding of the law of mathematics content. The generation of mathematics thought comes from the reasoning process of mathematical thinking, but not all of the mathematical thinking can eventually form a 
mathematical thinking. Mathematical method is to solve the mathematical problems in the process used in a variety of specific ways and means. Therefore mathematical thinking in strict sense is also different from the mathematical methods, people often referred to them as "mathematical thinking method", the reason is that the idea and the method of the two complement each other, closely related to each other. As a matter of fact, the method is the means to carry out the thought; the thought is the guidance in the course of the implementation of the concrete method. Generally speaking, when talking about mathematical thinking and methods to emphasize its guiding ideology, the focus on mathematical thinking; if the emphasis on specific implementation process, then focuses on the mathematical method. In fact, it also implies a corresponding method of thinking, and it also extends to the idea of the guiding role of the method. In this paper, it is discussed that the method of thinking and the method of the transformation is the. The inherent pattern of the idea of human thinking, which is always hoping that, the solution to the original knowledge and skills to solve the new problems, to change the status quo in. Of the idea is derived from the wisdom of life, but also widely used in mathematics, mathematics is made of the idea of the theoretical sublimation, it is also the core and many other ways of thinking, have the irreplaceable position in mathematics, has shown an important role[2].

\section{The teaching condition of realizing the thought of change}

Cultivation of consciousness. (1) carefully designed to resolve reasonable topic suggestions, to cultivate students' consciousness to the mathematical problem solving: Polka is the world banner, he knows the mathematics of the unknown to the known, simplified, the new method is easy, the old universal significance. How to solve the problem? How to induce inspiration? In Polka's view, the process of solving the problem is to change the course of the subject. He said: "the correct way of solving the right depends on the choice depends on from close to his direction to attack the fort, in order to identify which one thought is correct, which direction can tap into it, will be tempted with direction and ideas, to change the subject. (2) the principle of clear transformation, grasp the transformation strategy: mathematics is an organic whole, its various parts are interconnected, interdependent, mutual penetration. In order to form a three-dimensional space we arranged in a crisscross pattern, in the study of math problems, often use these links to change the problem properly, let it become simple, familiar with the problem. To complete the transformation, must first clear the general principle and method of transformation, and to consolidate the practice through typical examples. (3) the cultivation of students' association thinking, improve the transformation ability: Lenovo is a new thinking. When solving a problem, Lenovo is the basic method of our thinking, learning new knowledge, and the knowledge about the old Lenovo, can improve the efficiency of learning, in the process of solving problem, Lenovo from one problem to another problem is the basic way to realize the transformation, thus from thinking of migration, also can carry on the innovation of thinking. Mathematical problem solving process in a sense is known and unknown knowledge association process, through association to find out the relationship between the old and new knowledge, so as to find the solution to the idea [3].

The application of the thought of Transformation Though in University algebra. In college algebra, the use of the transformation of the thought of transformation of the examples is everywhere. Although the solution of equation, the equation of different types, different forms, and methods also has their own characteristics, but its basic idea is to transform, the basic approach is the elimination, reduction time, which is the unknown knowledge into knowledge, knowledge into easy difficult knowledge and complex knowledge into simple knowledge. We usually will be converted to rational irrational equation transformation equation, fractional equation to integral equation, equation to low equation transformation, called to a multiple element equation transformation, so that the problem becomes easy to be solved. In the above transformation, is the same requirements before and after deformation equation, this time the need to use the same solution of equivalent transformation principle, it is worth noting that, in consideration of all the factors, don't ignore the relationship between them. In the discussion of the nature of the function, the basic function of the image can be 
translated or symmetric transformation can also be a function of the analytical formula to get the answer. The transformation of the function of the visual function is transformed into the basis of image transformation, and it also shows that the image transformation is transformed into the unity of function analytic transformation. Algebraic and trigonometric, geometric transformation is also reflected in the various aspects of. The geometric problem can be transformed into a complex number by using the geometric meaning of the complex number, and the maximum value of the function of the geometric background is given by using the structure of algebra. This is equivalent to the transformation, the requirements of the transformation before and after the problem is fully necessary, so that the results obtained before and after the transformation is consistent. In addition, there are a number of non equivalence transformations, such as the use of the method of inequality in the proof of inequality. The non equivalence transformation only needs to find the sufficient condition for the conclusion of the original title, so that the process of reasoning and argument can be simplified. There are two times when we solve the inequality, often translated into solutions corresponding to one yuan and two times the equation has the root of the problem and there are several problems. These changes can make the problem simple, easy and convenient.

The application of the thought of the transformation to the University geometry. The generation of the thought of geometry, its history is profound. It can be said that every proposition is a certain geometric proposition before it gets through deductive reasoning, which is the premise of the proposition as deduction by its previous proposition conclusion, this has been attributed to some initial proposition does not prove so far. Geometry is a deductive science; there are axioms in its system - conclusions B- conclusions A- conclusions C... We can pass this conclusion even simple problem solving process, so that greatly improve the speed of problem solving. Do not need every problem from the original proposition, as long as the need to solve the problem to the conclusion of a link on the line. University of geometry from the study of the basic graphics in the plane geometry, complex graphics are through the transformation of the simple graph to solve, the three-dimensional geometry is often attributed to the plane geometry to solve. For example, the triangle is a simple basic graphics in the plane geometry, in the study of the triangle on the basis of the nature, for the study of quadrilateral and polygon can be transformed into a triangle to study. Angle and polygon transformation for the interior angles of a triangle and etc [4].

\section{Analysis of general principles of Transformation Though}

Simple principle. It is the key point to find the correct transformation method and the goal of turning back to the mathematics teaching and learning. But at the same time, it should be recognized that the system of mathematics knowledge is huge and changeable. So the process of the idea in the specific implementation will not be universal absolute unity mode, the need for thinking and strain problems vary, using their own knowledge system as the basis to solve the problem. However, after a lot of experience, it is found that it can be found in the dynamic changes in a number of useful principles. A mathematical problem may be some problems in a certain rule combination, when trying to solve such a problem when people first comprehensive attempt to understand its structure, for the complex structure which we will strive to make towards simplistic transformation. This is the simple principle that we should follow in the mathematical problem solving. This simple can be understood as a single point of knowledge, a simple expression of the form of expression or simple calculation method of processing.

Realization of specialization or generalization. Specialized or generalized realization of the so-called general is to solve a problem, the first solution than the general problem, and then the special, so as to obtain the solution of the original problem. General strategy is helpful to the promotion of proposition; on the other hand, it is also an effective way to solve many mathematical problems. Compared to the "general", the "special" problem is often simple, intuitive and specific, and easier to solve. Therefore, people have difficulty in solving mathematical problems of a general, often think of the first starting from solving the special cases and then the method to solve the special case of the general problem or result up application or extended to, so as to obtain the general 
solutions to the problem. For a specific problem, using the general strategy or is using a special strategy, on the one hand depends on the characteristics of the problem, such as a problem itself is special, and easy to be extended to the general situation, you can use the general strategy, if a problem is more general than their own, and can be regarded as the number of a relatively simple problem of combination, can use the special strategy on the other hand, the learners' thinking habits are decided on the same problem with what strategies, such as a problem can not only use the general strategy of return, also can use special strategy of return, some people are accustomed to some special. People are more accustomed to the general. Of course, no matter what kind of strategy to use, as long as the problem can be achieved, the final solution to the problem, it should be counted as an effective way. "Non equivalence" and "equivalence special" are two kinds of common classification. "Non equivalent specialization" refers to the special and not will solve the original problem completely to solve a new problem is only to solve the original problem play a supporting role, namely the original problem and new problems are not equivalent. The "equivalent specialization" is a new problem which is obtained after the specialization. The solution of the original problem can be solved completely, that is, the original problem is equivalent to the new problem.

Experimental research. China's education has always attached great importance to the traditional ideas and methods, in recent years, with the promotion of the new curriculum reform, the ideological method of teaching has been clearly defined as the task of teaching. The specific skills teachers in the teaching of problem-solving, the general method of problem solving questions focus on the classification, contempt; despise way of thinking from the summary; quickly shows the intention to solve problems, ignoring the inquiry study of students. The students in the study is more "to understand, but not to do" long-term confusion. All these problems should be attributed to the neglect of teaching and learning. Fortunately, the problem is changing, as a front-line educator, the author is willing to study and practice the teaching of ideas and methods in practical work.

Experimental summary. After the experiment, students basically clear the essence that is integrated to the thinking of the problem as simple problems; the new problems to the familiar problem; a problem to be solved to convert another problem; convert a form into another form of the problem. Many students can take the initiative to use the method of thinking in solving problems, mainly in learning new knowledge is not passive but active contact wait and accept his own knowledge and system integration of new knowledge and to establish the relation table now see the new problems or is no longer thought comprehensive problem where but careful analysis, analysis try to figure out, consider before has done the related questions, whether the subject is special or general performance; strengthen confidence in solving the problems of the idea, is not a panacea, but under the guidance of the analysis of the direction of the problem although there will still be can not solve the problem but they believe that with my knowledge the system can further enrich the smell provided the ability of solving problems[5].

\section{Summary}

Mathematics teaching in the University, to the thinking of the idea that everywhere, so important, so in the future of the University as a mathematics teacher, in particular, in the teaching process, not only to impart the knowledge of students, more important is to permeate the mathematics method. Whether it is teaching, or problem solving, we all want to be familiar with the new knowledge into the knowledge, the complex into simple knowledge, make the teaching process smoothly, students gain knowledge in the relaxed teaching situation, grasp the skills. The practice shows that if teachers can attach importance to the thought of mathematics education, play an important role in mathematical thought and method in mathematics teaching, can cultivate students' innovative spirit and application ability, an important way to improve the comprehensive quality of students. In college mathematics teaching, teachers win side show the occurrence and development of the process of knowledge, and try to penetration to the thinking of students, cultivate students' ability to use of the idea, give full play to the guiding role of the idea of the method. This is conducive to the students to develop a good 
quality of thinking, to implement quality education by Qi, is conducive to the cultivation of their innovative ability, and helps them to develop a good habit of finding problems to solve problems.

\section{References}

[1] Chen Honglian. Application of the idea in mathematics teaching of [J]. China school education. 2011 (17).

[2] Zhu Liu. The application of thinking in the middle school mathematics [J]. Shanghai middle school mathematics. 2007 (12).

[3] Zhang Taiming. A discussion on the way of thinking and the application of [J]. Technology in mathematics teaching. 2007 (20).

[4] Liu Zhigang. The use of the transformation of the idea to answer questions for example [J]. Mathematics teaching and communication. 2007 (01).

[5] Sun rain. Journal of transforming thought in mathematics application [J]. Jining teachers college. 2002 (04). 